\title{
Porous photoresist stamps for selective plasma treatment
}

\author{
U. Stöhr ${ }^{1}$, A. Dohse ${ }^{2}$, P. Hoppe ${ }^{1}$, A. Gehringer ${ }^{1}$, M. Thomas ${ }^{2}$, C.-P. Klages ${ }^{2}$, H. Reinecke ${ }^{1}$ \\ ${ }^{1}$ Laboratory for Process Technology, IMTEK, University of Freiburg, Freiburg, Germany \\ ${ }^{2}$ Fraunhofer Institute for Surface Engineering and Thin Films IST, Braunschweig, Germany
}

\begin{abstract}
A porous plasma stamp has been developed allowing selective plasma treatment and coating of flat surfaces using dielectric barrier discharges at atmospheric pressure. It is shown that flat substrate surfaces can be activated in the same scale as the cavity dimensions and that the treated areas are defined by the footprint of the cavities with a precision in micrometer scale.
\end{abstract}

Keywords: area-selective surface treatment, dielectric barrier discharge, plasma printing

\section{Introduction}

Area-selective plasma treatment at atmospheric pressure has gained increased interest for industrial applications e. g. in bioscience. Atmospheric pressure plasma processes are attractive due to short process times and potential low costs as no vacuum equipment is required. There are different approaches to treat surfaces selectively at atmospheric pressure. A well known technique is the plasma jet, $[1,2]$, another one is the so-called "plasma printing" process $[3,4]$. In this process a plasma stamp, a device with cavities in which the plasma is burning, is pressed on the surface of the substrate that should be plasma treated.

In this work a plasma stamp with a new gas inlet approach was developed. It is based on a porous sinter metal electrode that is covered with photoresist.

\section{Plasma Stamp Design}

The plasma stamp consists of a porous, planar sinter metal that is used as high voltage electrode and that serves as carrier for the photo resist. A photo resist is applied on the metal and structured there to form cavities. This stamp is pressed on the substrate which is connected to a planar and grounded metal electrode on its other side. Fig. 1 shows the stamp design in the setup.

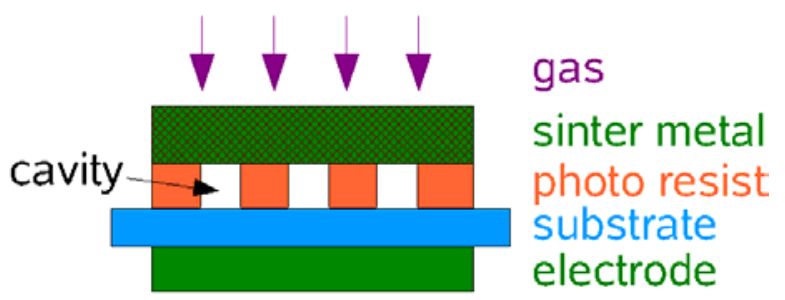

Fig. 1: Design concept of selective plasma treatment using a porous plasma stamp.

To treat substrates selectively at the locations given by the cavities, a flat stamp surface of roughness below $2-3 \mu \mathrm{m}$ is required. Otherwise the plasma would burn in the resulting gap between the stamp and the substrate, outside the cavities. To obtain this flatness, the sinter metal was specially machined as explained in sec. 4.

\section{Materials}

The chosen porous material was the stainless steel type AISI $316 \mathrm{~L}$ with an effective pore size of $14 \mu \mathrm{m}$ and a plate thickness of $5 \mathrm{~mm}$. This sinter metal is resistant to an oxidizing environment up to a temperature of $500{ }^{\circ} \mathrm{C}$.

The used resist was TMMF S2055 (Tokyo Ohka Kogyo (TOK)), a high-resolution, laminatable photo resist with a thickness of $55 \mu \mathrm{m}$. The resist reaches aspect ratios of $4.7: 1$ (height: width) and is stable against high voltages, temperatures up to $150{ }^{\circ} \mathrm{C}$, and is chemically inert. The resist properties and applications were investigated in [5]. The used substrate material was $75 \mu \mathrm{m}$ thick, biaxially oriented polypropylene (BOPP) foil.

\section{Manufacturing Process}

The delivered sinter metal plates were first milled to have rectangle pieces with a size of $90 \times 80 \mathrm{~mm}$. To prevent high electric fields, their edges were rounded. The resulting plates were cleaned consecutively with acetone, isopropanol, and finally with deionized water. To avoid a gap between the stamp and the substrate, the sinter metal plates need to have a very flat surface. Experiences from previous works, [6], showed that the waviness of the surface must be below $5 \mu \mathrm{m}$ on a length of $5 \mathrm{~cm}$. Several tests lead to the conclusion that this can only be achieved starting with at least $5 \mathrm{~mm}$ thick sinter metal. One side of the metal plates was treated using wire electrical discharge machining (WEDM). To treat the plates the machine Robofil 2020 SI (Charmilles) and a wire with a diameter of $250 \mu \mathrm{m}$ was used. In a first step the wire was routed over the plate with a certain distance. In the second and third WEDM step the wire was positioned in each step 
$50 \mu \mathrm{m}$ closer to the backside of the plate. Finally two more steps were performed without changing the wire distance to polish the surface. The result is shown in Fig. 2. The WEDM process creates small corroded metal particles that close the sinter metal pores of the machined surface. To remove them, the metal plates were finally anodic electro-cleaned.

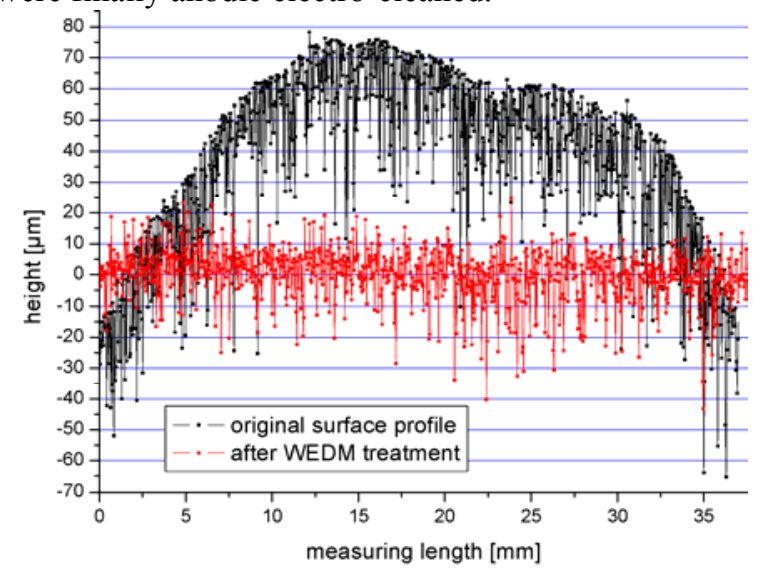

Fig. 2: WEDM treatment result.

The photo resist is delivered between two protective foils. After removing of the first foil the resist was laminated on the sinter metal using the laminator model "Riston Laminator" (DuPont). For the lamination rubber coated rolls, a lamination speed of $0.7 \mathrm{~m} / \mathrm{s}$, and a pressure of about 2 bar were used. The laminator bottom roll had a temperature of $73.5^{\circ} \mathrm{C}$, the top roll $57.5^{\circ} \mathrm{C}$.

After removing the second protective foil, the resist was exposed using light with a wavelength of $365 \mathrm{~nm}$ (I-line emission of mercury) with a dose of $270 \mathrm{~mJ} / \mathrm{cm}^{2}$ using a mask aligner MA-6 (Süss Microtec). Afterwards the stamp was put on a hotplate that was preheated to $50{ }^{\circ} \mathrm{C}$. The temperature was then increased to $60{ }^{\circ} \mathrm{C}$ and held for $5 \mathrm{~min}$ to cross-link the resist. Finally a hard bake step followed at $150{ }^{\circ} \mathrm{C}$ for $35 \mathrm{~min}$. At this step the unexposed parts of the resist that were not cross-linked are drawn into the metal but the sinter metal has such a large surface that this resist doesn't close its pores. Therefore it is not necessary to develop the resist after the hard bake.

\section{Results}

\subsection{Selective Plasma Enhanced Coating}

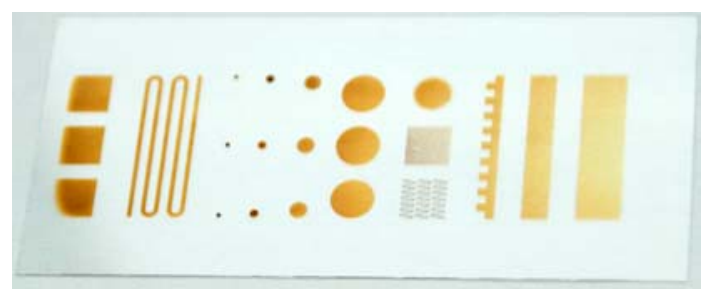

Fig. 3: Selectively plasma-coated $100 \mu \mathrm{m}$ thick glass
The aim of the developed plasma stamp is to be able to coat flat surfaces selectively with a simple setup. To test the resulting coating performance, a mixture of $1 \%$ acetylene and $99 \%$ helium was applied to the backside of the stamp. The gas diffused through the sinter metal to the stamp cavities where the plasma was burning. There the acetylene is polymerized and deposited on the substrate. Fig. 3 and $\mathbf{4}$ show first coating results. It can be seen that is possible to coat the surface selectively using an atmospheric pressure PECVD process. The high resolution of the plasma printing process is demonstrated in Fig. 4, where a grid is shown in detail. The grid bars have a width of $50 \mu \mathrm{m}$ proving that the coating is selective down to micrometer scale.

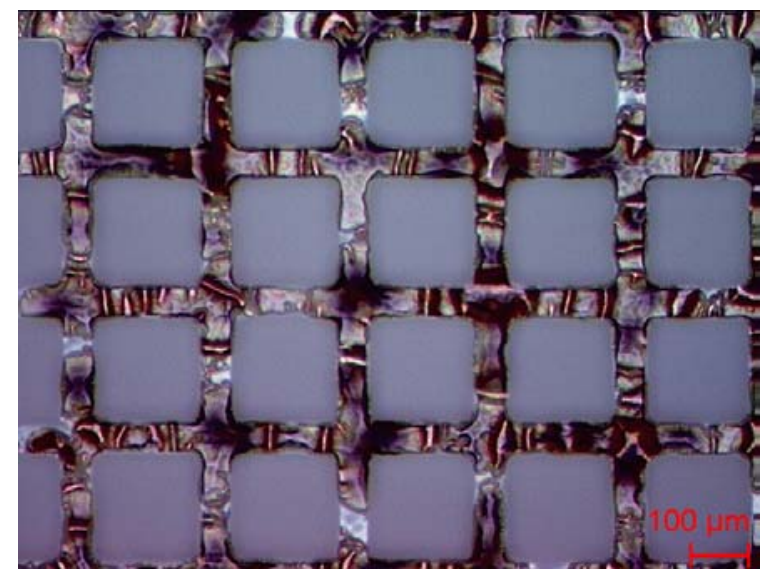

Fig. 4: Detail of the slide shown in Fig. 3.

Fig. 4 visualizes that the structure of the sinter metal, will be transferred to the surface of the coating. This leads to rough surfaces as shown in Fig. 5. This surface profile is the coating result of the comb-like structure from Fig. 3 after 2 min of plasma coating using a complete process gas flow of $7.5 \mathrm{slm}$, with the above mentioned gas mixture. Measurements with a surface profiler show that the coated "comb teeth" have a width of $990 \pm 10 \mu \mathrm{m}$.

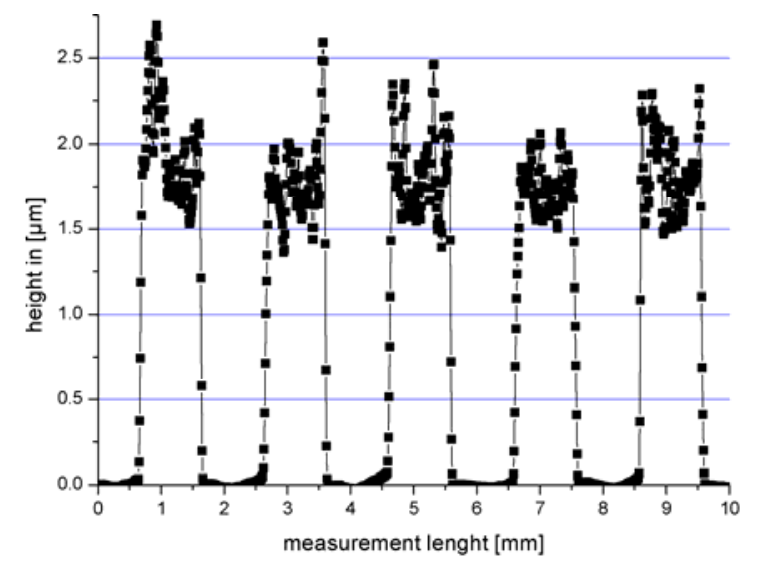

Fig. 5: Surface profile of a coated comb-like structure. 


\subsection{Selective Amination by Plasma Coating}

The selective amination of the BOPP surface obtained by plasma coating was investigated using fluorescence labeling. BOPP foils were coated for times of $5-40 \mathrm{~s}$ using a mixture of $10 \mathrm{slm}$ nitrogen, one time with a mass fraction of $0.08 \%$ (3-aminopropyl)trimethoxysilane (APTMS), and with a mass fraction of $1.14 \%$ 1,2-diaminocyclohexane (DACH). The coated foils were labeled with fluorescamine, a coupling agent that forms fluorescent products only with primary amines [7].

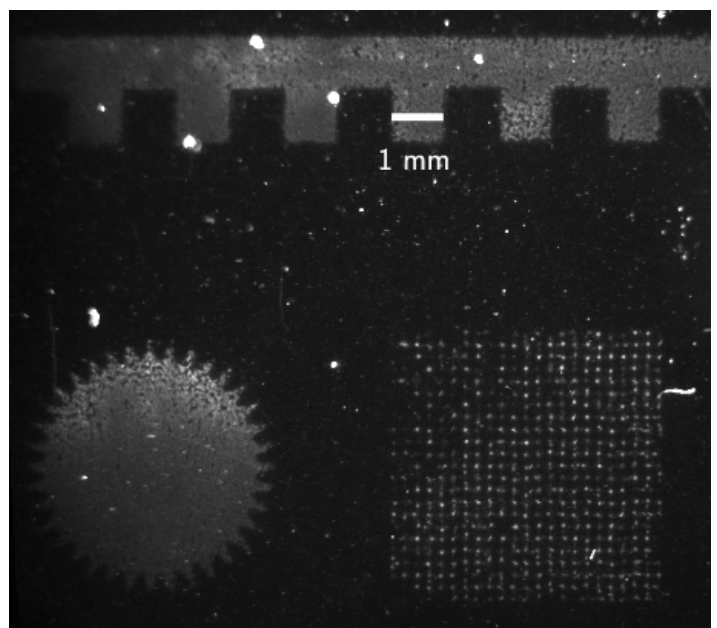

Fig. 6: Fluorescence image of a BOPP foil coated with $\mathrm{DACH}$ and labeled with fluorescamine. The comb-like structure has teeth with a width of $1 \mathrm{~mm}$ as tagged in the image. The structure on the lower right is a grid structure consisting of lines with a width of $50 \mu \mathrm{m}$ and space of $175 \mu \mathrm{m}$ between them.

Fluorescence images of coated foils were produced using the biochip reader model Biodetect 645/4 (Fraunhofer IPM). The excitation wavelength was $395 \pm 15 \mathrm{~nm}$, and the detection wavelength $460 \pm 20$ nm. Fig. 6 shows the result of a foil coated for $5 \mathrm{~s}$ using DACH. The fluorescence light was amplified by a factor 4 for a better visualization of the result. Using APTMS or DACH coatings with primary amino groups were created on the BOPP surface during the plasma process. The "comb teeth" structure shown in Fig. 6 has a width of $1.000 \pm 0.003 \mathrm{~mm}$ in the stamp. In the fluorescence images we measured a width of $0.977 \pm 0.054 \mathrm{~mm}$ at the tagged position. This is in consistency to the results of sec. 5.1. The accuracy of the width is more imprecisely as the above reported surface profiler measurements since the surface profiler has a better resolution than the biochip reader.

Fig. 6 shows that the $50 \mu \mathrm{m}$ wide lines of the grid structure couldn't be completely aminized in comparison to the acetylene coating demonstrated in Fig. 4. In addition also images of unlabeled foils were made si- multaneously. The fluorescence intensity in the coated, labeled areas is by a factor of 3 larger than on the coated, but unlabeled reference. The reason for the detection of fluorescence light at the unlabeled coated reference is the light scattering at the coated areas, which will be detected by the biochip reader although it is no fluorescence light.

\section{Conclusions}

A new plasma stamp for area-selective plasma treatment using dielectric barrier discharge at atmospheric pressure was developed and tested. It can be used to activate, functionalize and coat flat substrates surfaces area-selectively down to micrometer scale. The coated layers show a high surface roughness making the process suitable when large reactive surfaces are required. Using suitable process gases it is also possible to generate functional chemical groups like primary amines.

\section{References}

[1] H. Tan, T. Akagi, and T. Ichiki, Journal of Photopolymer Science and Technology 19, 245 (2006).

[2] R. Brandenburg, J. Ehlbeck, M. Stieber, T. v. Woedtke, J. Zeymer, O. Schlüter, and K.-D. Weltmann, Contrib. Plasma Phys. 47, 72 (2007).

[3] C. Penache, C. Gessner, T. Betker, V. Bartels, and C.-P. Klages, IEE Proc.-Nanobiotechnol., vol. 151, pp. 139 - 144, 2004.

[4] C.-P. Klages, A. Hinze, K. Lachmann, C. Berger, J. Borris, M. Eichler, M. von Hausen, A. Zänker and M. Thomas, Plasma Process. Polym. 4, 208 (2007).

[5] U. Stöhr, P. Vulto, P. Hoppe, G. Urban, and H. Reinecke, J. Micro/Nanolith. MEMS MOEMS 7, 033009 (2008).

[6] U. Stöhr, A. Dohse, P. Hoppe, M. Thomas, K. Kadel, C.-P. Klages, H. Reinecke, Plasma Process. Polym. 6, 228 (2009).

[7] S. Udenfriend, S. Stein, P. Böhlen, W. Dairman, W. Leimgruber and M. Weigele, Science 178, 871 (1972). 